ОСОБЕННОСТИ ВИЗУАЛИЗАЦИИ МЕТАСТАТИЧЕСКОГО ПОРАЖЕНИЯ МОЛОЧНОЙ ЖЕЛЕЗЫ ПРИ ВЫПОЛНЕНИИ ГИБРИДНОЙ ТЕХНОЛОГИИ ОДНОФОТОННО-ЭМИССИОННОЙ КОМПЬЮТЕРНОЙ ТОМОГРАФИИ, СОВМЕЩЕННОЙ С КОМПЬЮТЕРНОЙ ТОМОГРАФИЕЙ, РЕДКИЙ КЛИНИЧЕСКИЙ СЛУЧАЙ

\author{
Тарасов Н.И., Дризнер Е.А., Шевченко С.А., Белых Д.В.
}

ГАУЗ СО «Свердловский областной онкологический диспансер», Екатеринбург, е-таіl:

Tarasov.nick2010@yandex.ru

В статье представлено описание редкого клинического случая - метастатического поражения молочной железы из перстневидно-клеточной карциномы желудка у пациентки с клинической картиной воспаления, лимфостаза верхней конечности и молочной железы, ранее перенесшей субтотальную дистальную резекцию желудка по Ру с лимфодиссекцией, установленным метастатическим поражением костей. По данным литературного обзора, экстрамаммарные метастазы в молочную железу составляют около 0,5-2,0\% среди всех раков молочных желез, а в структуре метастазов желудка составляют лишь 0,3\%. В процессе постановки диагноза авторы объединили и совместно оценивали данные маммографического, ультразвукового, магнитно-резонансного методов, а также гибридного метода радионуклидной диагностики - однофотонно-эмиссионной компьютерной томографии, совмещенной с рентгеновской компьютерной томографией (ОФЭКТ/КТ). Ключевую роль в диагностике имели результаты иммуногистохимического анализа, подтвердившего метастатический генез поражения молочной железы. Как правило, метастазы карциномы желудка в молочную железу встречаются в более раннем возрасте, чаще с левой стороны, в данном случае атипичны и возраст пациента, и сторона поражения. Подобных наблюдений в отечественных и международных источниках опубликовано около 60. Данный клинический случай демонстрирует особенности визуализации метастатического поражения молочной железы при проведении ОФЭКТ/КТ.

Ключевые слова: перстневидно-клеточный рак, метастаз, рак молочной железы, ОФЭКТ/КТ.

\title{
CASE REPORT: MULTIMODAL DIAGNOSTIC APPROACH IN METASTATIC LESION OF BREAST FROM A SIGNET-RING CELL CARCINOMA OF THE STOMACH, ROLE OF HYBRID SPECT/CT TECHNOLOGY
}

\author{
Tarasov N.I., Drizner E.A., Shevchenko S.A., Belyikh D.V.
}

GAUZ SO «Sverdlovsk regional oncologic hospital», Yekaterinburg, e-mail: Tarasov.nick2010@yandex.ru

This article presents a rare clinical case of metastatic lesion of breast from a signet-ring cell carcinoma of the stomach in a patient with a clinical manifestation of inflammatory (diffuse) type of right breast cancer. Studies review indicates that breast metastases from extra-mammary malignancies occur in $0,5-2 \%$ among all breast cancers and in $0,3 \%$ among gastric cancer metastases. We have summarized and collaboratively assessed the information that we have gathered: a brief history of a patient and important findings with details of mammographic, ultrasound, MR investigations and in addition, taking in account our clinic's opportunities relatively new hybrid method of radionuclide diagnostics single-photon emission computed tomography with computed tomography (SPECT-CT) of breast and regional lymphatic nodes. The results of the immunohistochemical analysis played the key role in diagnostics and determined the metastatic origin of breast lesion. This is notable, given the fact that gastric adenocarcinoma metastases usually occur in younger patients and have left-sided localization, in this case both age and side are atypical. We have found approximately 60 published cases of similar secondary metastatic lesions of breast in various international medical resources. Thus, this case report demonstrates the importance of multimodal diagnostic approach and collaborative work of health professionals in management of a patient with atypical results of clinical, radiological, laboratory investigations. Keywords: signet-ring cell carcinoma, metastasis, breast, breast cancer, SPECT/CT.

Несмотря на успехи в развитии скрининга и лечения рака молочной железы, данное заболевание является распространенным и все еще остается лидирующей причиной в 
структуре женской смертности как в России [1], так и в мире [2]. Однако экстрамаммарные метастазы в молочную железу довольно редки, составляют всего 0,5-2,0\% среди всех раков молочных желез, а среди метастазов рака желудка - лишь 0,3\% [3]. Наиболее частыми источниками маммарных метастазов являются меланома, лимфома, рак легкого, рак яичников, мягкотканные саркомы, а также гастроинтестинальные опухоли и опухоли мочеполовой системы $[1,3]$.

При метастатическом поражении молочной железы данные клинических методов могут имитировать воспалительные процессы или воспалительный рак: отек, болезненность, покраснение [4].

По данным Lee и коллег [3], средний интервал между установлением первичной болезни и метастатическим поражением молочных желез составляет 1,25 месяца.

Первым методом в алгоритме диагностики при подозрении на рак молочной железы является УЗИ с оценкой регионарных лимфоузлов. В крупном метаанализе Rupali Sood и иные обнаружили чувствительность УЗИ 80,1\% (95\% ДИ, 72,2-86,3\%), специфичность - 88,4\% (95\% ДИ, 79,8-93,6\%) [3, 4].

Tan и коллеги [5] сообщают о превосходстве УЗИ ввиду низкой чувствительности маммографии - 49\%, при хорошей специфичности последней - 89\%. В то же время маммография имеет лучшую специфичность у женщин старше 50 лет. Так, по данным этого же исследования, 20\% раков, обнаруженных на УЗИ, были не видны при проведении маммографии, также по данным маммографии и УЗИ обнаружили характерные для метастатического поражения молочной железы радиологические паттерны - отсутствие достоверной визуализации образований и микрокальцинатов.

Мультипараметрическая магнитно-резонансная томография (МРТ) молочных желез имеет превосходные показатели чувствительности и специфичности в отношении раннего выявления рака, а также в качестве уточняющего метода диагностики второй линии, однако содержит долю ложноположительных результатов за счет контрастирования доброкачественных образований [6].

ОФЭКТ/КТ применяется в сомнительных случаях при проведении вышеописанных методов диагностики, а также на фоне плотных молочных желез, при установленном раке для выявления статуса регионарных лимфоузлов. Данный метод не может являться методом диагностики первой линии ввиду немалой лучевой нагрузки, достигающей 8-10 мЗв. Отдельный вид исследования - сцинтимаммография с использованием специализированной камеры - применяется для скрининга при плотных молочных железах [7].

Задача исследования: оценить особенности гибридного метода - однофотонной эмиссионной компьютерной томографии, совмещенной с рентгеновской компьютерной 
томографией (ОФЭКТ/КТ), в мультимодальной диагностике метастатического поражения молочной железы из перстневидно-клеточной карциномы у пациентки с отеком верхней конечности и воспалением молочной железы справа.

\section{Материалы и методы исследования}

Пациентка 67 лет, перенесшая в 2017 г. субтотальную дистальную резекцию желудка по Ру с лимфодиссекцией по поводу злокачественного новообразования тела желудка /pT2/pN1/G3/R0/ стадия IIA, 5 адьювантных курсов полихимиотерапии (ПХТ), в июне 2020 г. обратилась с жалобами на отек верхней конечности, воспаление молочной железы. По данным позитронно-эмиссионной томографии в 2020 г. были выявлены метастазы в кости. Дифференциальная диагностика проводилась между диффузной формой рака молочной железы и лимфомой кожи со вторичным поражением молочной железы. Инструментальная диагностика включала рентгеновскую маммографию (РМГ), ультразвуковое исследование (УЗИ), магнитно-резонансную томографию (МРТ), гибридное радионуклидное исследование - ОФЭКТ/КТ молочных желез и регионарных лимфоузлов. Окончательный диагноз был установлен по результатам патоморфологического и иммуногистохимического исследований препарата после диагностической секторной резекции молочной железы.

По результатам маммографии достоверно дополнительных образований не обнаружено, справа определялся диффузно усиленный и деформированный структурный рисунок, без микрокальцинатов. В косой проекции в верхнем квадранте определялся более плотный участок перестройки структуры 12х7 мм (в анамнезе трепан-биопсия). Кожная полоска диффузно инфильтрирована, сосок втянут, BI - RADS 4c. Слева определялась умеренно выраженная фиброгландулярная ткань молочной железы без микрокальцинатов и патологических изменений.

По данным УЗИ на фоне умеренно выраженной фиброзной ткани сохранялся отек ткани правой молочной железы, у соска кнаружи определялась гипоэхогенное образование 16x9 мм с выраженным кровотоком. В аксиллярной зоне лимфоузлы не были изменены, нечетко определялись межсекторальные лимфоузлы 9-10 мм.

По результатам МРТ в правой молочной железе наряду с утолщением дермы выявлялись множественные участки накопления контрастного вещества (КВ) без четких контуров, что было интерпретировано в пользу диффузной формы рака. Также выявлена интрамаммарная лимфаденопатия без признаков поражения подмышечных лимфоузлов.

Следующим этапом пациентке была выполнена чрескожная толстоигольная полифокальная пункция объемного образования правой молочной железы и произведена автоматическая трепан-биопсия опухоли под УЗ-контролем. Биопсийный материал был направлен на гистологическое и ИГХ-исследования, по результатам которых в объеме 
трепанобиоптата молочной железы был определен фиброз с единичными протоками без атипии и фрагментами неизмененного покровного плоского эпителия.

Учитывая несоответствие данных маммографического, ультразвукового и магнитнорезонансного методов диагностики результатам гистологического заключения, а также нарастание клинических проявлений в виде гиперемии, отека правой молочной железы, развития лимфедемы правой верхней конечности, консилиумом было принято решение о проведении ОФЭКТ/КТ молочных желез и регионарных лимфоузлов.

Исследование выполнялось на аппарате General Electric Discovery NM/CT 670 с туморотропным радиофармпрепаратом Технетрил ${ }^{99 \mathrm{~m}} \mathrm{Tc}$, (МИБИ) с активностью 520 МБк. Препарат вводился в дорсальную вену левой стопы, сканирование выполнено через 15 минут после инъекции РФП. На 1-м этапе выполнялся сбор ядерных данных с использованием низкоэнергетических коллиматоров высокого разрешения (LEHR), угол вращения $180^{\circ}$ для каждого детектора, матрица 128х128, 12 секунд/кадр. 2-й этап выполнялся непосредственно после окончания 1-го этапа, КТ-сканирование выполнено по низкодозовому протоколу (100 $\mathrm{kV}, 50$ mAs, время сканирования 12,6 секунды, толщина среза 2,5 мм). Для измерения интенсивности накопления РФП был использован полуколичественный индекс «очаг/фон», отражающий соотношение счета над патологическим очагом и фоном.

При проведении исследования на ОФЭКТ/КТ-сканах в стандартных проекциях определялось физиологическое накопление РФП в щитовидной железе, миокарде, печени, мышцах. Кожа правой молочной железы была диффузно утолщена, вблизи соска обнаружен интрадермальный очаг с патологическим повышенным накоплением РФП, индекс «очаг/фон» составил 2,3. В пределах молочной железы определялись множественные образования с нечеткими неровными контурами, на границе наружных квадрантов выявлен очаг с патологической гиперфиксацией РФП, при измерении был получен индекс «очаг/фон», равный 3,0, что было интерпретировано в пользу злокачественного процесса. В левой молочной железе патологического накопления РФП обнаружено не было. В регионарных лимфоузлах значимого накопления РФП не обнаружилось (индекс «очаг/фон» 1,4), структурно подлопаточный лимфоузел справа имел частично утраченную дифференцировку, при этом максимальный размер по короткой оси не превышал 8 мм. Дополнительно в зоне сканирования визуализировалось множество склеротических очагов в грудных позвонках.

Таким образом, по данным ОФЭКТ/КТ (рис. 1) были найдены нетипичные для рака молочной железы изменения - очаг гиперфиксации РФП в утолщенной дерме при отсутствии значимого накопления в регионарных лимфоузлах (рис. 2), что в целом охарактеризовало диагностический метод ОФЭКТ/КТ как наиболее чувствительный в данной клинической ситуации (рис. 3). 

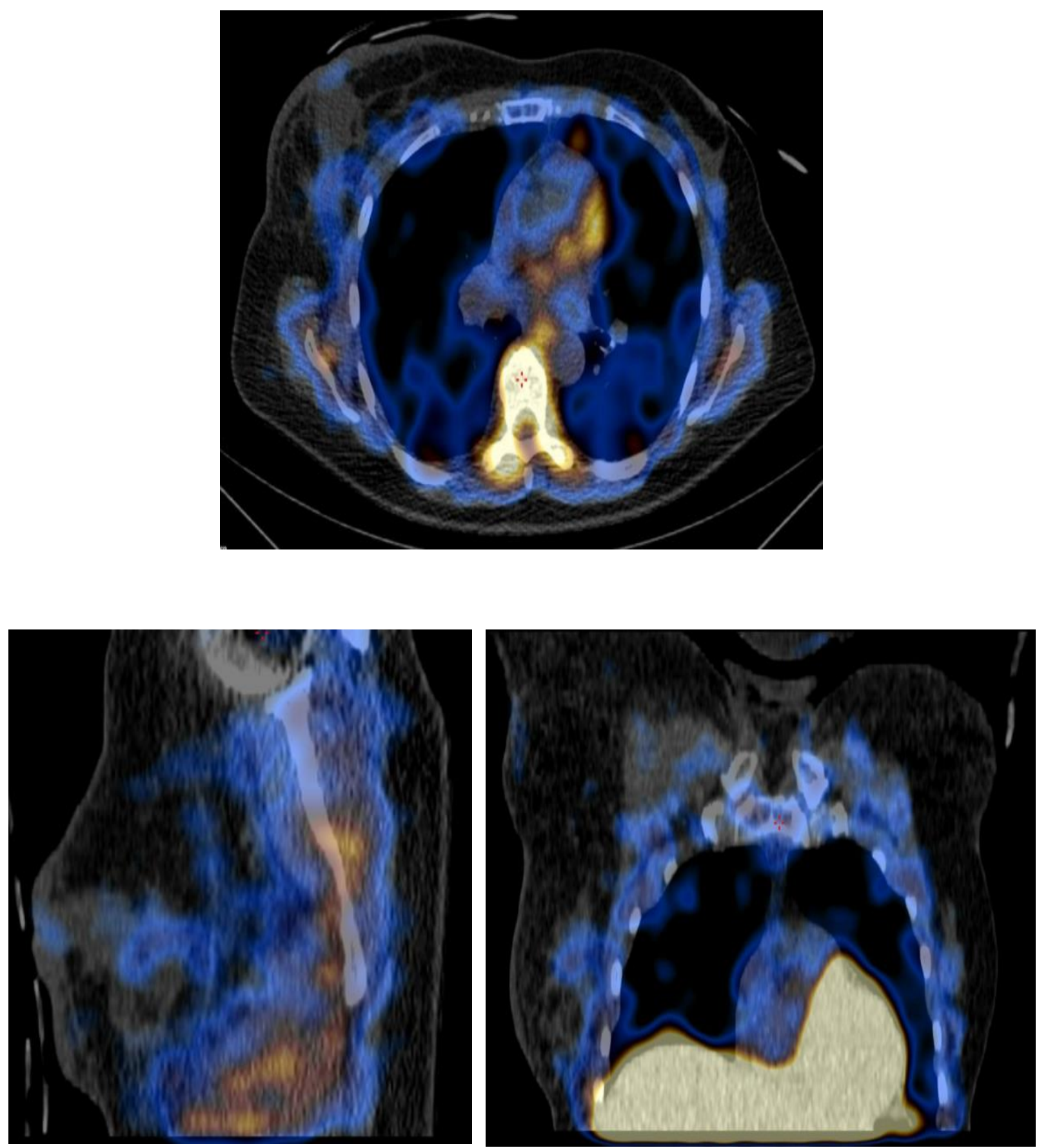

Рис. 1. ОФЭКТ/КТ молочных желез и регионарных лимфоузлов с Технетрилом, меченым ${ }^{99 m} T c$, совмещенные изображения в аксиальной, сагиттальной и корональных плоскостях соответственно (сверху вниз)

Пациентке выполнена диагностическая секторальная резекция правой молочной железы. По данным гистологического исследования верифицирован рак молочной железы с перстневидноклеточной дифференцировкой, диффузным характером роста, лимфоваскулярной и периневральной инвазией, далее материал направлен на иммуногистохимическое исследование, по результатам которого выявлена иммуноморфоструктура вторичного очага перстневидноклеточной аденокарциномы желудка: 
экспрессия цитокератинов 7 и 20 при отсутствии экспрессии эстрогеновых и прогестероновых рецепторов, высокий индекс пролиферативной активности, Кi67 - 90\%, сомнительная реакция Her2/neu дообследована FISH-методом, трактована как ложноположительная.

Определяется крупный очаг гиперфиксации туморотропного РФП на границе наружных квадрантов правой молочной железы, также выявлено очаговое повышенное накопление РФП в утолщенной дерме вблизи соска. Признаков лимфаденопатии не обнаружено. Значимого накопления в регионарных лимфоузлах не отмечено.
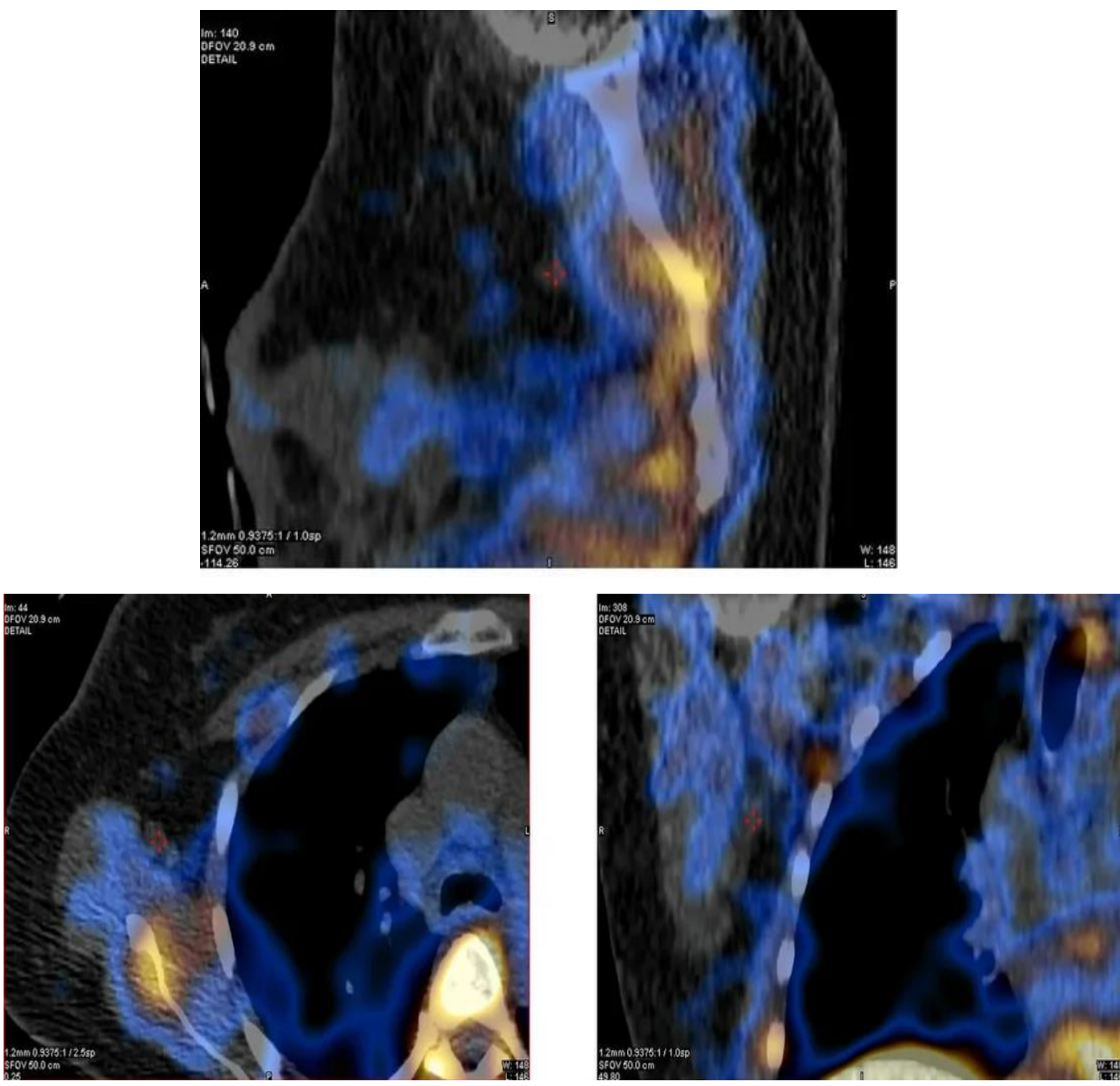

Рис. 2. ОФЭКТ/КТ молочных желез и регионарных лимфоузлов с Технетрилом, меченым ${ }^{99 m} T c$, совмещченные изображения в аксиальной, сагиттальной и корональных плоскостях соответственно (сверху вниз)

Кпереди от широчайшей мышцы спины определяется подмышечный лимфатический узел с частично утраченной структурой, максимальный размер по короткой оси не более 8 мм, 
накопление РФП на уровне фона, данных за патологическое накопление в регионарных лимфоузлах не получено.

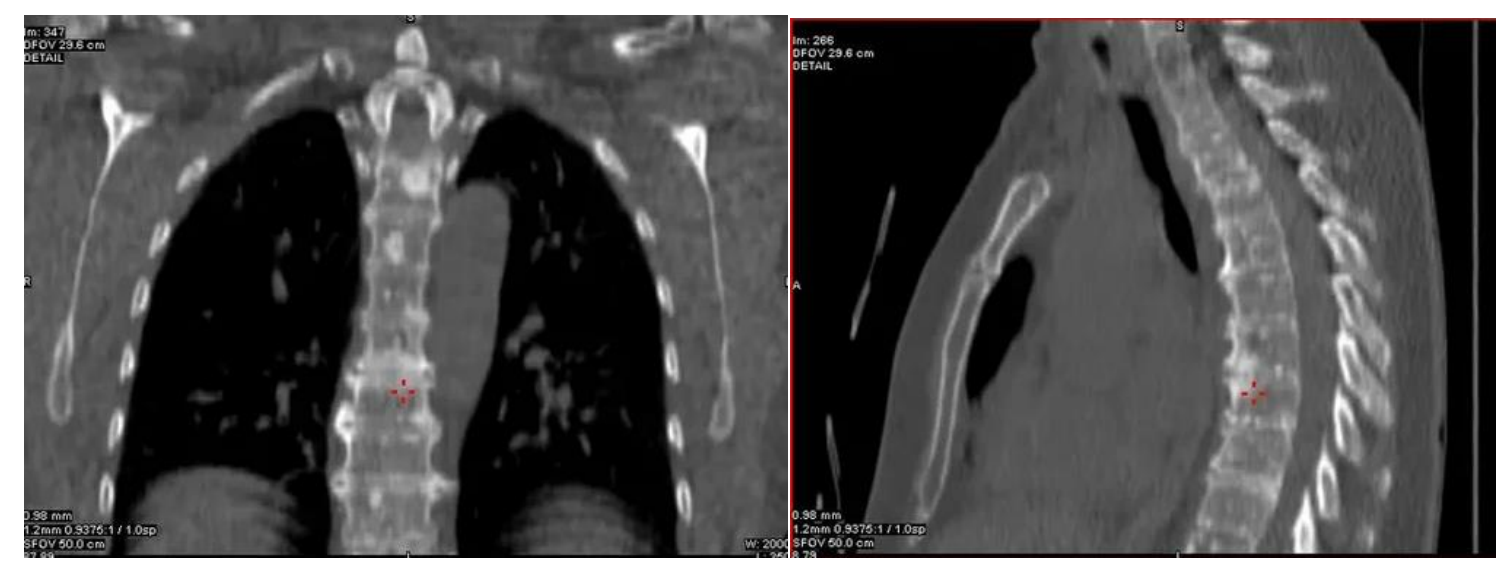

Рис. 3. КТ-скань, 2-й этап ОФЭКТ/КТ, реконструкиии в корональной и сагиттальных плоскостях соответственно

На фоне дегенеративных изменений грудного отдела позвоночника в виде боковых и передних остеофитов, субхондрального склероза в грудных позвонках выявлены множественные склеротические полиморфные очаги. В лопатках и ребрах патологические изменения достоверно не визуализируются.

\section{Результаты исследования и их обсуждение}

На основании атипичных клинических симптомов (отек молочной железы и верхней конечности с признаками поражения кожи) авторами был заподозрен неопластический процесс в дерме со вторичным поражением молочной железы. По данным ОФЭКТ/КТ молочных желез и регионарных лимфоузлов, помимо повышенного патологического накопления радиофармпрепарата (РФП) в самой молочной железе, гиперфиксация была выявлена и в утолщенной дерме, что позволило предположить вторичное поражение молочной железы. После диагностической секторной резекции при патоморфологическом и иммуногистохимическом исследованиях препарата обнаружен рак молочной железы и дермы c перстневидноклеточной дифференцировкой, диффузным характером роста, лимфоваскулярной и периневральной инвазией, экспрессией цитокератинов 7 и 20 при отсутствии экспрессии эстрогеновых и прогестероновых рецепторов. Данные изменения были интерпретированы как вторичный очаг аденокарциномы желудка.

Согласно данным литературы, доля злокачественных образований молочной железы, не являющихся первичными, составляет всего 0,5-2,0\% в клинической практике и 1,7-6,6\% при аутопсии. В метаанализе средний возраст пациентов при вторичном поражении молочных желез составил 45 лет, при первичном раке - 61 год. Как правило, метастазы аденокарциномы желудка в молочную железу встречаются в более раннем возрасте, чаще с левой стороны. 
Несмотря на вышеперечисленное, в нашем наблюдении пациент принадлежит к более старшей возрастной группе, имея при этом нетипичную для рака желудка сторону поражения. Атипичными симптомами также явились отек молочной железы и верхней конечности, отсутствие достоверных данных за образования и микрокальцинаты по данным маммографии, обнаружение очага гиперфиксации РФП в коже молочной железы, отсутствие признаков поражения регионарных лимфоузлов, экспрессия опухолевыми клетками цитокератинов 7 и 20 типа в отсутствие экспрессии ER и PR, сомнительная реакция HER2/neu (2+) была дообследована FISH-методом, трактована как ложноположительная. Подобных наблюдений в отечественных и международных источниках опубликовано около 60. Таким образом, в диагностике метастатического поражения молочной железы необходимо принимать во внимание атипичные данные методов диагностики (клинических, радиологических и лабораторных).

Мультимодальный и междисциплинарный подход к диагностике позволяет принять наиболее правильное решение в плане тактики дальнейшего лечения.

\section{Заключение}

В данном наблюдении авторами продемонстрированы атипичные клинические и радиологические симптомы при метастатическом поражении молочной железы у пациентки с раком желудка.

\section{Список литературы}

1. Гажонова В.Е., Ефремова М.П., Дорохова Е.А. Современные методы неинвазивной лучевой диагностики рака молочной железы // РМЖ. 2016. № 5. С. 321-324.

2. Bray F., Ferlay J., Soerjomataram I., et al. Global cancer statistics 2018: GLOBOCAN estimates of incidence and mortality worldwide for 36 cancers in 185 countries. CA Cancer J. Clin. 2018. Vol. 68. P. 394-424.

3. Lee S.K., Kim W.W., Kim S.H., Hur S.M., Kim S., Choi J.H., Cho E.Y., Han S.Y., Hahn B.K., Choe J.H. Characteristics of metastasis in the breast from extramammary malignancies. J. Surg Oncol. 2010. Vol. 101. P. 137-140. DOI: 10.1002/jso.21453.

4. Rupali Sood, Anne F. Rositch, Delaram Shakoor, Emily Ambinder. Ultrasound for Breast Cancer Detection Globally: A Systematic Review and Meta-Analysis. Journal of Global Oncology. 2019. No 5. P. 57-62. DOI: 10.1200/JGO.19.00127.

5. Tan K.P., Mohamad Azlan Z., Rumaisa M.P., Siti Aisyah Murni M.R., Radhika S., Nurismah M.I., Norlia A., Zulfiqar M.A. The comparative accuracy of ultrasound and mammography in the detection of breast cancer. Med. J. Malaysia. 2014. Vol. 69 (2). P.79-85. 
6. Breast MRI: State of the Art. Ritse M. Mann, Nariya Cho, Linda Moy. Radiology 2019. Vol. 292. P. 520-536. DOI: 10.1148/radiol.2019182947.

7. Kinoshita T., Fukui N., Anan K., Iwamoto T., Niikura N., Kawai M., Hayashi N., Tsugawa K., Aogi K., Ishida T. Comprehensive prognostic report of the Japanese Breast Cancer Society Registry in 2004. Breast Cancer. 2016. Vol. 23. P. 39-49. DOI: 10.1007/s12282-015-0644-5. 